\title{
Maximum likelihood estimation of aggregated Markov processes
}

\author{
FENG QIN, ANTHONY AUERBACH And FREDERIGK SAGHS \\ Department of Biophysical Sciences, State University of New York at Buffalo, Buffalo, New York 14214, USA
}

\section{SUMMARY}

We present a maximum likelihood method for the modelling of aggregated Markov processes. The method utilizes the joint probability density of the observed dwell time sequence as likelihood. A forward-backward recursive procedure is developed for efficient computation of the likelihood function and its derivatives with respect to the model parameters. Based on the calculated forward and backward vectors, analytical formulae for the derivatives of the likelihood function are derived. The method exploits the variable metric optimizer for search of the likelihood space. It converges rapidly and is numerically stable. Numerical examples are given to show the effectiveness of the method.

\section{INTRODUGTION}

We consider a finite state, continuous time Markov process. The complete process is not observable, rather the state space is partitioned into classes, and it is only possible to observe which class the process is in. Such Markov processes have been termed aggregated Markov processes, and have found considerable application in the modelling and analysis of single ion channel currents that occur in certain neurophysiological investigations (see, for example, Colquhoun \& Hawkes 1995).

The theoretical properties of aggregated Markov processes have been extensively studied in the literature, both to determine what properties of the underlying Markov process can be deduced from the observable aggregated process and also to aid the validation of proposed ion channel gating mechanisms (see, for example, Colquhoun \& Hawkes 1982; Fredkin \& Rice 1986; Ball \& Sansom 1988). Here we focus on the computational aspect. Our objective is to estimate the model parameters of the underlying Markov process from the observable aggregated process.

The prime difficulty in modelling aggregated Markov processes is the ambiguity arising from the aggregation of the underlying state space into observation classes. It has been shown that this ambiguity is even more serious than that caused by the observation noise (Fredkin \& Rice 1992). In this work we will deal with the ambiguity from state aggregation, assuming no observation noise. We will also ignore the effects of missed events due to the finite detector bandwidth.

When each state can be observed distinctly, the aggregated Markov process reduces to a traditional Markov process. For the latter, it is well known that the distribution of the dwell times in a given state follows a single exponential. This property can be extended to aggregated Markov processes. In general, the probability density for the lifetime of an aggregated
Markov process in a given class is a multi-exponential, with as many components as states in that class. Consequently, some aspects of the underlying Markov process can be inferred by constructing the lifetime histograms of the observed dwell times and then fitting these histograms with sums of exponentials. This has been the traditional histogram fitting technique for single-channel data analysis (Colquhoun \& Sigworth 1995). It is conceptually simple and has been used extensively. However, it has several disadvantages. For example, it requires a relatively large amount of data, otherwise the histogram may be inaccurate to approximate the lifetime distribution. More importantly, it does not include the cross-correlation information between adjacent dwell times. As a consequence, conversion of the histogram-fitting results to the underlying model parameters may be impossible due to incomplete information.

More recently, the maximum likelihood approach has gained increasing popularity because of its associated optimality. Pioneering studies in this area by Horn \& Lange (1983) yielded an algorithm that fits a model to the data set directly, without the need to construct the histograms. Their method was based on the discretization of the continuous Markov process (although they also suggest the use of continuous dwell time distributions). The likelihood was defined as the joint probability of all discrete samples. A procedure based on probability recursions was developed for practical evaluation of the likelihood. This procedure may be considered as a variant of the forward algorithm for hidden Markov modelling (Rabiner 1989) in the case of clean observations. Maximization of the likelihood is performed by a nonlinear regressive technique where the required partial derivatives are calculated numerically. A major drawback of their method is the requirement of considerable computing time and a large memory space, mainly due to the discretization of the underlying continuous process. 
More efficient likelihood methods have been explored by several authors (Chay 1988; Ball \& Sansom 1989). These methods deal with the continuous Markov process directly without discretization, and exploit the dwell times of the process as observations rather than the data samples. The likelihood function is defined as the joint probability density of the observed dwell time sequence (Fredkin \& Rice 1986). Because there are many fewer dwells than samples, the evaluation of the dwell time likelihood function has a significantly reduced computational requirement as compared with the likelihood function defined over discrete samples. The general dwell time likelihood method implemented by Ball \& Sansom (1989) is applicable to both binary channels and channels with multiple conductance levels. However, the method relies on numerically calculated derivatives for the search of the likelihood surface, which slows the computation because an accurate evaluation of a derivative requires multiple evaluations of the likelihood function (Press et al. 1992). This problem becomes more serious as the number of model parameters increases.

The purpose of this work is to advance a general and efficient maximum likelihood procedure for modelling of aggregated Markov processes. As before, the method utilizes the joint probability density of the observed dwell time series as likelihood. By fully exploiting the structure of the likelihood function, a forwardbackward recursive procedure is developed for efficient computation of the likelihood function and its derivatives with respect to model parameters. This forwardbackward procedure has a similar spirit to that used in hidden Markov modelling (Rabiner 1989), but is conceptually different. Based on the calculated forward and backward vectors, analytical formulae for the derivatives of the likelihood function are derived. Our method makes use of a variable metric optimizer (Press et al. 1992) for searching the likelihood surface. Besides its rapid convergence, this optimizer also has the advantage of generating a good approximation to the curvature of the likelihood surface at the maximum point, thus allowing the parameter error estimates to be calculated relatively easily. Because analytical derivatives are used, the method is fast, taking a few seconds for a multistate model with thousands of events.

\section{AGGREGATED MARKOV PROGESSES}

The theory of aggregated Markov processes has been extensively studied, mainly in the context of ion channel modelling (see, for example, Colquhoun \& Hawkes 1982; Fredkin \& Rice 1986). We describe here only those aspects essential to understanding the maximum likelihood estimation. It should be pointed out that most results in the literature have been specialized, covering the case of only two aggregates, primarily due to the fact that the early findings on biological channels showed only two conducting states, closed and open. Fortunately, the extension of these results to the general case is fairly straightforward. Here we present the results with an arbitrary number of aggregates.
Let $X(t), t \geqslant 0$ be a stationary Markov process in continuous time with a finite number of states. Label the states $1,2, \ldots, N$. The transitions between these states are governed by a transition rate matrix, $Q$, in which the off-diagonal elements, $q_{i j}$, are the transition rates between states $i$ and $j$, and the diagonal elements are defined so that the rows sum to zero. The forward equation for $X(t)$ is

$\frac{\mathrm{d} P(t)}{\mathrm{d} t}=P(t) Q$,

with the initial condition $P(0)=\boldsymbol{I} . P(t)$ is known as the transition probability matrix, the $(i, j)$ th element of which defines the probability of the process being in state $j$ at time $t$ given that it was in state $i$ at time 0 . The solution to the forward equation may be represented by

$P(t)=\exp (Q t)$,

where the matrix exponential is defined in the usual manner (see, for example, Bellman 1960).

$X(t)$ is not completely observable. The state space is aggregated into $M(M \leqslant N)$ observation classes, and it is only possible to observe which class the process is in. Suppose there are $n_{a}$ states in class $a$. Throughout the paper, we denote the observation classes by the letters $a, b, c$, etc. In the context of ion channel modelling, a class refers to the states with the same current. One of the most important results in the theory of aggregated Markov processes is an analytical formula for the probabilities of the transitions confined within a class. To state the formula, we partition the $Q$-matrix into

$Q=\left[\begin{array}{cccc}Q_{11} & Q_{12} & \cdots & Q_{1 M} \\ Q_{21} & Q_{22} & \cdots & Q_{2 M} \\ \vdots & \vdots & \ddots & \vdots \\ Q_{M 1} & Q_{M 2} & \cdots & Q_{M M}\end{array}\right]$,

where, for example, $Q_{a b}$ is the submatrix of the transition rates between the states of classes $a$ and $b$. Then, the transitions for $X(t)$ to stay within a class, $a$, for a given duration, $t$, and then exit into another class, $b$, have probability densities given by the following matrix (Colquhoun \& Hawkes 1982):

$G_{a b}(t)=\exp \left(Q_{a a} t\right) Q_{a b}$,

where the $(i, j)$ th element of this matrix is the conditional probability that $X(t)$ enters class $a$ from its $i$ th state, remains in the class throughout the time interval $[0, t]$ and then exits into the $j$ th state of class $b$.

The observations for $X(t)$ consist of a series of dwell times during which $X(t)$ is in a given class $a$ for a time $t_{1}$, then in a class $b$ for a time $t_{2}$, etc. A fundamental description of such dwell times is the so-called onedimensional distribution, $f_{a}(t)$, i.e. the probability density of a given dwell time, $t$, in class $a$. Colquhoun \& Hawkes (1982) have derived $f_{a}(t)$ in the case of two observation classes. As generalized to multiple classes, their results may be stated as

$f_{a}(t)=\pi_{a} \exp \left(Q_{a a} t\right) \sum_{b \neq a}\left(Q_{a b} \mathbf{1}\right)$ 
where $\pi_{a}$ is a row vector of the equilibrium probabilities for the process to enter the states in class $a$, and the $\mathbf{1}$ denotes any column vector of ones of appropriate length. That is, the probability density of a dwell time, $t$, in a class, $a$, is obtained by the entry probabilities into that class, post multiplied by the probabilities of the transitions occurring within that class, and post multiplied by the probability densities of leaving that class. The summation in the last term takes into account the uncertainty of the next class in the process.

In this study, we assume that $X(t)$ is at thermodynamic equilibrium. Such an assumption yields several important consequences. For example, the equilibrium probabilities of $X(t)$, denoted by $w_{j}, j=1$, $2, \ldots, N$, may be obtained via solution of the detailed balance equations:

$w_{i} q_{i j}=w_{j} q_{j i}, \quad i, j=1,2, \ldots, N$.

The entry probability vector, $\pi_{a}$, is given by (Colquhoun \& Hawkes 1982)

$\pi_{a}=\frac{\sum_{b \neq a} w_{b} Q_{b a}}{\sum_{b \neq a} w_{b} Q_{b a} 1}$,

where $w_{b}$ is a row vector of the equilibrium probabilities of the states in class $b$. Another consequence of the equilibrium assumption is that the $Q$ matrix, as well as its submatrices $Q_{a a}$, are ensured to be diagonalizable and that the corresponding eigenvalues are all strictly negative except one equal to 0 for $Q$ (see, for example, Fredkin et al. 1985; Ball \& Sansom 1988). Obviously, such properties are desirable from a computational point of view, as discussed in the next section.

Taking one step further, Fredkin \& Rice (1986) showed that the one-dimensional distribution (equation (5)) may be extended to consecutive dwell time pairs, and even more generally, to any series of dwell times. To state their results, let $\boldsymbol{t}=t_{1} t_{2} \ldots t_{L}$ be the list of time intervals and $\boldsymbol{a}=a_{1} a_{2} \ldots a_{L}$ the list of corresponding classes. Then, the joint probability density of such a dwell time series is given by

$f(\boldsymbol{t}, \boldsymbol{a})=\pi_{a_{1}} G_{a_{1} a_{2}}\left(t_{1}\right) G_{a_{2} a_{3}}\left(t_{2}\right) \cdots G_{a_{L} a_{L+1}}\left(t_{L}\right) \mathbf{1}$,

where $a_{L+1}$ refers to the subset of the states that do not belong to $a_{L}$. That is, the joint probability density is given by the vector of the entry probabilities into class $a_{1}$, post multiplied by the matrix of the probability densities of transitions within class $a_{1}$ for a duration, $t_{1}$, and then exit into class $a_{2}$, post multiplied by the matrix of probability densities of transitions within class $a_{2}$ for a duration, $t_{2}$, and then exit into class $a_{3}$, and so on.

When viewed as a function of the model parameters, equation (8) is the likelihood of observing the dwell time series $\boldsymbol{t}$ and $\boldsymbol{a}$ given the model. To exhibit the model dependence, we denote the likelihood function by $f(\theta)$ where $\theta$ designates the unknown transition rates in the $Q$ matrix. Maximization of $f(\theta)$ with respect to $\theta$ yields the maximum likelihood estimates of the model parameters for a given data set. These estimates are assured to be asymptotically unbiased and maximally efficient in terms of the information content (Cox \& Miller 1965).

\section{MAXIMUM LIKELIHOOD ESTIMATION}

We have seen that the likelihood of an observed dwell time sequence is a complicated function of the underlying model parameters. In this section, we show how to efficiently evaluate this function as well as its derivatives with respect to the model parameters. We also describe the optimization method for search of the likelihood space. Finally, we discuss how to assess the error estimates of the estimated parameters.

\section{(a) Forward and backward recursions}

Equation (8) shows that the likelihood of a series of dwell times consists of the product of the probability density matrices of individual dwell times. This suggests that the practical calculation of the likelihood function may be implemented recursively. Specifically, we define the forward vectors, $\alpha_{k}$, as

$\alpha_{k}^{T}=\pi_{a_{1}} \prod_{j=1}^{k} G_{a_{j} a_{j+1}}\left(t_{j}\right)$,

i.e. the forward partial product of the likelihood function from the beginning to the $k$ th dwell time. Then we can solve for $\alpha_{k}$ inductively by forward recursions

$\alpha_{k}^{T}=\alpha_{k-1}^{T} G_{a_{k} a_{k+1}}\left(t_{k}\right), \quad k=1,2, \cdots, L$,

where the initial condition $\alpha_{0}^{T}=\pi_{a_{1}}$. The final result of this recursion gives the likelihood as

$f(\theta)=\alpha_{L}^{T} 1$,

i.e. the sum of the components of the last forward vector, $\alpha_{L}$.

In a similar manner, we can define the backward vectors, $\beta_{k}$, as:

$\beta_{k}=\prod_{j=k}^{L} G_{a_{j} a_{j+1}}\left(t_{j}\right) \mathbf{1}$,

i.e. the backward partial product of the likelihood function from the end to the $k$ th dwell time. Again we can solve for $\beta_{k}$ inductively by backward recursions

$\beta_{k}=G_{a_{k} a_{k+1}}\left(t_{k}\right) \beta_{k+1}, k=L, L-1, \ldots, 1$,

where the initial condition $\beta_{L+1}=\mathbf{1}$.

Strictly speaking, only the forward vectors, $\alpha_{k}$, are required for calculating the likelihood, as seen from equation (11). The backward vectors, $\beta_{k}$, however, are needed for calculating the derivatives of the likelihood function, as shown below.

The physical meaning of the forward and backward vectors is easily visualized. The forward vector, $\alpha_{k}$, has the dimension equal to the number of the states in class $a_{k}$ and its $i$ th component, $\alpha_{k}(i)$, represents the probability density of the partial dwell time series from 
the beginning to the $k$ th dwell and then exiting into the $i$ th state of class $a_{k+1}$. Another simple interpretation of $\alpha_{k}$ is the vector of entry probabilities for the $(k+1)$ th sojourn. Similarly, the backward vector, $\beta_{k}$, has the same dimension as $\alpha_{k}$, and the $i$ th component, $\beta_{k}(i)$, corresponds to the probability density of the partial dwell time series from the $k$ th dwell to the end, given the starting state being the $i$ th state of class $a_{k}$.

The overall computation involved in the forward and backward recursions can be estimated approximately as follows. Each calculation of $G_{a b}(t)$ takes about $n_{a}\left(\epsilon+n_{a}+n_{a} n_{b}\right)$ multiplications, where $\epsilon$ is the number of multiplications needed for an exponential operation. Multiplying $G_{a b}(t)$ by a previous forward or backward vector takes another $n_{a} n_{b}$ multiplications. Thus, each recursion takes a total number of $n_{a}\left(\epsilon+n_{a}+n_{b}+n_{a} n_{b}\right)$ multiplications. Suppose the dwell pair ' $a b$ ' occurs $l_{a b}$ times in the observed dwell time sequence. Then the total amount of the calculation involved in the forward or backward recursions is approximately

$\sum_{a \neq b} l_{a b} n_{a}\left(\epsilon+n_{a}+n_{b}+n_{a} n_{b}\right)$.

Obviously, this number not only varies with the model size and data length, but also with the model structure and the dwell time distributions. In the case of $m=2$, which is often encountered in ion channel modelling, there are only two possible dwell pairs, either $C O$ or $O C$, thus $l_{a b}=l_{b a}=L / 2$, and the above estimate may be simplified as

$\left(n_{c} n_{o} n+n^{2}+n \epsilon\right) L / 2$

which is proportional to the data length and approximately quadratic in the number of states.

Finally, readers familiar with the literature on hidden Markov models may notice the similarity between the forward-backward procedure described here and that used in hidden Markov modelling (Rabiner 1989). Indeed, they have the same spirit and motivation. However, they are also conceptually different. The forward-backward procedure in the hidden Markov modelling performs recursions over individual samples, while the recursion here is over dwells. Moreover, the underlying processes are different: one is the hidden Markov chain in discrete time, and the other is the aggregated Markov process in continuous time.

\section{(b) Spectral representation}

The probability density matrix $G_{a b}(t)$ is involved in each forward and backward recursion. Thus, the efficiency on its calculation has a direct effect on the efficiency of the overall procedure. Fortunately, there exists an efficient way for its calculation. This is accomplished by making use of the spectral representation of $Q_{a a}$. In this approach, we decompose the $Q_{a a}$ matrix into

$Q_{a a}=\sum_{i=1}^{n_{a}} \lambda_{a, i} A_{a, i}$ where $\lambda_{a, i}$ is the $i$ th eigenvalue of $Q_{a a}$, and $A_{a, i}$ is a matrix determined from the corresponding left and right eigenvectors, satisfying

$\sum_{i=1}^{n_{a}} A_{a, i}=I$

and

$A_{a, i} A_{a, j}= \begin{cases}A_{a, i} & \text { if } i=j \\ 0 & \text { otherwise. }\end{cases}$

Note that the eigenvalues $\lambda_{a, i}$ are all strictly negative, as a consequence of the reversibility of $X(t)$. Substituting equation (16) into equation (4) and then making use of equations (17) and (18), yields

$G_{a b}(t)=\left[\sum_{i=1}^{n_{a}} A_{a, i} \exp \left(\lambda_{a, i} t\right)\right] Q_{a b}$.

This equation implies that $G_{a b}(t)$ may be calculated from the spectral decomposition results of $Q_{a a}$ through plain matrix addition and multiplication. The quantities $\lambda_{a, i}$ and $A_{a, i}$ do not change with $t$, thus they need to be evaluated only once for a given set of parameter values.

\section{(c) Derivatives of likelihood function}

We now show how to calculate the derivatives of the likelihood function with respect to the model parameters. We assume the initial probability $\pi_{a_{1}}$ is known, so it is independent of the model parameters. Experiments suggest that with long records, the estimation results are insensitive to the choice of the initial probabilities as long as they are made consistent with the first dwell class. This insensitivity may be attributed to the fact that the effect of the initial probabilities decays exponentially with time.

Applying the chain rule for differentiating matrix products to equation (8) and then making use of equations (9) and (12), we obtain

$\frac{\partial f}{\partial Q}=\sum_{k=1}^{L} \frac{\partial}{\partial Q}\left[\alpha_{k-1}^{T} G_{a_{k} a_{k+1}}\left(t_{k}\right) \beta_{k}\right]$

where $\alpha$ and $\beta$ are viewed as constant vectors, so the differentiation in the summand is only with respect to the $G$ matrix.

Equation (20) suggests that the key to calculate $\partial f / \partial Q$ is to calculate the derivatives of the following expression:

$\alpha^{T} G_{a b}(t) \beta$,

where $\alpha$ and $\beta$ are appropriate vectors. Note that only the submatrices $Q_{a a}$ and $Q_{a b}$ are involved in expression (21). Thus we only need to calculate its derivatives with respect to the elements of these two submatrices. As $G_{a b}(t)$ depends linearly on $Q_{a b}$, the derivative to $Q_{a b}$ is relatively easy; a straightforward application of matrix differentiation rules yields

$$
\begin{aligned}
\frac{\partial}{\partial Q_{a b}}\left[\alpha^{T} G_{a b}(t) \beta\right] & =\left[\alpha^{T} \exp \left(Q_{a a} t\right)\right]^{T} \beta^{T} \\
& =\sum_{i=1}^{n_{a}} A_{a, i}^{T} \alpha \beta^{T} \exp \left(\lambda_{a, i} t\right) .
\end{aligned}
$$


To derive the derivative to $Q_{a a}$, we notice the following equality (Ball \& Sansom 1989):

$\frac{\partial \exp \left(Q_{a a} t\right)}{\partial x}=\sum_{i=1}^{n_{a}} \sum_{j=1}^{n_{a}} A_{a, i} \frac{\partial Q_{a a}}{\partial x} A_{a, j} \gamma_{a, i, j}(t)$,

where $x$ is an arbitrary variable, and $\gamma_{a, i, j}(t)$ is a scalar function of $t$ defined by

$\gamma_{a, i, j}(t)=\left\{\begin{array}{cc}t e^{\lambda_{a, i} t} & i=j \\ \frac{e^{\lambda_{a, i} t^{t}-e^{\lambda_{a, j} t}}}{\lambda_{a, i}-\lambda_{a, j}} & i \neq j .\end{array}\right.$

From equation (23) it follows that

$\frac{\partial}{\partial x}\left[\alpha^{T} G_{a b}(t) \beta\right]=\sum_{i=1}^{n_{a}} \sum_{j=1}^{n_{a}} \alpha^{T} A_{a, i} \frac{\partial Q_{a a}}{\partial x} A_{a, j} Q_{a b} \beta \gamma_{a, i, j}(t)$.

Taking $x$ as an element of $Q_{a a}$ and writing the subsequent derivatives in matrix form, we derive

$\frac{\partial}{\partial Q_{a a}}\left[\alpha^{T} G_{a b}(t) \beta\right]=\sum_{i=1}^{n_{a}} \sum_{j=1}^{n_{a}} A_{a, i}^{T} \alpha \beta^{T} Q_{a b}^{T} A_{a, j}^{T} \gamma_{a, i, j}(t)$.

This equation, together with equation (22), provide the derivatives of expression (21) with respect to the elements of the $Q$ matrix.

We now go back to equation (20). By the use of the formulae (22) and (26), we arrive at

$\begin{aligned} \frac{\partial f}{\partial Q_{a b}} & =\sum_{i=1}^{n_{a}} A_{a, i}^{T}\left[\sum_{\substack{k=1 \\ a_{k}=a_{a_{k+1}=b}}}^{L} \alpha_{k-1} \beta_{k}^{T} \exp \left(\lambda_{a, i} t_{k}\right)\right] \\ \frac{\partial f}{\partial Q_{a a}} & =\sum_{i=1}^{n_{a}} \sum_{j=1}^{n_{a}} A_{a, i}^{T}\left[\sum_{\substack{k=1 \\ a_{k}=a}}^{L} \alpha_{k-1} \beta_{k}^{T} Q_{a a_{k+1}}^{T} \gamma_{a, i, j}\left(t_{k}\right)\right] A_{a, j}^{T} .\end{aligned}$

These two equations show that the derivatives of the likelihood function with respect to the elements of the $Q$ matrix may be calculated analytically from the forward and backward vectors as well as the spectral factors of the submatrices $Q_{a a}$. Evaluations of these two equations are straightforward. From equation (27) we see that only those dwell times at class $a$ with a successor at class $b$ are involved in the calculation of $\partial f / \partial Q_{a b}$. Similarly, equation (28) shows that only the dwell times at class $a$ are used in the calculation of $\partial f / \partial Q_{a a}$.

Finally, we notice that the derivatives given in equations (27) and (28) do not take into account the constraints that the sum of each row of $Q$ is equal to zero. This problem may be circumvented by considering the diagonal elements in $Q$ as functions of offdiagonal elements and then making use of the chain rule. That is, if $\partial f / q_{i j}, 1 \leqslant i, j \leqslant N$ are the derivatives obtained from equations (27) and (28), then the 'true' derivative of the likelihood $f$ with respect to the rate $q_{i j}$, $i \neq j$, after taking account of the constraint $\Sigma_{j} q_{i j}=0$, can be calculated by

$\frac{\partial f}{\partial q_{i j}}+\frac{\partial f}{\partial q_{i i}} \cdot \frac{\partial q_{i i}}{\partial q_{i j}}=\frac{\partial f}{\partial q_{i j}}-\frac{\partial f}{\partial q_{i i}}$,

where $\partial q_{i i} / q_{i j}=-1$. These corrected derivatives are used in optimization.

\section{(d) Maximization of likelihood}

We have described how to compute the likelihood and its derivatives. The next step is to maximize the likelihood with respect to the chosen parameters. This is essentially an optimization problem, and can be solved by a variety of methods. Perhaps the most powerful one among them is the variable metric method of Davidon, which uses the Fletcher-Powell approximation to the inverse of the Hessian matrix of second derivatives and an exact line search with adaptive step sizes (see, for example, Press et al. 1992). This optimizer has several advantages. First, it converges rapidly, and the convergence becomes quadratic when the parameters are sufficiently close to the maximum point. Second, a programmed version of the method exists in most numerical libraries, making it almost universally available. Finally, it has been successfully applied to solve a wide range of problems, demonstrating its practicability and reliability.

\section{(e) Parameter error estimates}

After obtaining the optimum estimates of the model parameters, it is necessary to assess the variability for these estimates. Approximate standard errors can be obtained from the curvature of the likelihood function at its maximum (Cox \& Miller 1965):

$\operatorname{Cov}(\theta) \approx H^{-1}(\theta)$,

where Cov is the covariance matrix, and $H$ is the Hessian matrix of second derivatives. The diagonal elements of the covariance matrix correspond to the variances of the estimated parameters.

The exact Hessian matrix can be calculated numerically, but involves intensive computing. A faster, although less accurate, way is to use directly the approximate inverse Hessian matrix generated by the optimizer. This is another reason why we chose the variable metric method for maximizing $f$. It can be shown that for a quadratic function of $n$ variables the matrices generated by the method converge to the correct inverse Hessian matrix after $n$ steps. Clearly, for a non-quadratic function this no longer holds. However, in the vicinity of a maximum point the likelihood function may usually be well approximated by a quadratic function. Thus we can still expect it to provide information about the local Hessian matrix at that point.

\section{IMPLEMENTATION}

The forward and backward recursions described in the previous section may run into numerical problems. Because the probability density matrix for a dwell time often has elements less than one, the forward vectors usually decay with increasing $k$ and the backward vectors with decreasing $k$. For sufficiently large $k$, the dynamic range of these vectors will exceed the precision range of essentially any machine. Fortunately, this problem can be avoided by ongoing scaling using a procedure similar to that in hidden Markov modelling (Rabiner 1989). 
Consider the computation of the forward vectors. For each $k, 0 \leqslant k \leqslant L$, we first compute $\alpha_{k}$ according to the induction formula (10), and then we multiply it by a scaling coefficient $c_{k}$,

$c_{k}=\frac{1}{\left\|\alpha_{k}\right\|}$,

where $\|\cdot\|$ represents the sum of the components of a vector. The scaled vector, denoted by $\bar{\alpha}_{k}$, is then used for computing the next $\alpha_{k+1}$. By induction we can write $\bar{\alpha}_{k}$ as

$\bar{\alpha}_{k}=\left(\prod_{i=0}^{k} c_{i}\right) \alpha_{k}$.

Setting $k=L$ and multiplying both sides by the unit vector $\mathbf{1}$, we have

$f=\left(\prod_{i=0}^{L} c_{i}\right)^{-1}$

or

$\log f=-\sum_{i=0}^{L} c_{i}$

Thus the log likelihood can be computed properly from the scaling coefficients.

Next we compute the $\beta$ vectors from the backward recursion. The only difference here is that we use the same scale factors for each $k, 1 \leqslant k \leqslant L$ for the $\beta$ s as was used for the $\alpha$ s. Then the scaled $\bar{\beta}_{k}$ can be written as

$\bar{\beta}_{k}=\left(\prod_{j=k}^{L} c_{j}\right) \beta_{k}$.

Multiplying equations (32) and (35) and then using the equality $\alpha_{k} \beta_{k}=f$, gives

$\bar{\alpha}_{k} \bar{\beta}_{k}=1$.

As $\left\|\bar{\alpha}_{k}\right\|=1$, this equation implies that using the same scaling factors on the $\beta$ s as was used on the $\alpha$ s is indeed an effective way of keeping the computation within reasonable bounds.

In terms of the scaled forward and backward vectors, the partial derivatives of the likelihood function given in equations (27) and (28) can be rewritten as

$$
\begin{aligned}
\frac{\partial f}{\partial Q_{a b}} & =\frac{1}{f} \sum_{i=1}^{n_{a}} A_{a, i}^{\tau}\left[\begin{array}{c}
\left.\sum_{\substack{k=1 \\
a_{k}=a_{a_{k+1}=b}}}^{L} \bar{\alpha}_{k-1} \bar{\beta}_{k}^{T} \exp \left(\lambda_{a, i} t_{k}\right)\right] \\
\frac{\partial f}{\partial Q_{a a}}
\end{array}=\frac{1}{f_{i=1}} \sum_{i=1}^{n_{a}} \sum_{j=1}^{n_{a}} A_{a, i}^{T}\left[\sum_{\substack{k=1 \\
a_{k}=a}}^{L} \bar{\alpha}_{k-1} \bar{\beta}_{k}^{T} Q_{a a_{k+1}}^{T} \gamma_{a, i, j}\left(t_{k}\right)\right] A_{a, j}^{T} .\right.
\end{aligned}
$$

If we choose to maximize the log likelihood, then

$$
\begin{aligned}
& \frac{\partial \log f}{\partial Q_{a b}}=\sum_{i=1}^{n_{a}} A_{a, i}^{T}\left[\sum_{\substack{k=1 \\
a_{k}=a_{a_{k+1}=b}}}^{L} \bar{\alpha}_{k-1} \bar{\beta}_{k}^{T} \exp \left(\lambda_{a, i} t_{k}\right)\right] \\
& \frac{\partial \log f}{\partial Q_{a a}}=\sum_{i=1}^{n_{a}} \sum_{j=1}^{n_{a}} A_{a, i}^{T}\left[\sum_{\substack{k=1 \\
a_{k}=a}}^{L} \bar{\alpha}_{k-1} \bar{\beta}_{k}^{T} Q_{a a_{k+1}}^{T} \gamma_{a, i, j}\left(t_{k}\right)\right] A_{a, j}^{T} .
\end{aligned}
$$

As the $\bar{\alpha}$ and $\bar{\beta}$ vectors have been properly scaled, the right-hand sides of the above equations can be computed without any numerical problem. Thus, the scaling of the forward and backward vectors provides a means for the direct evaluation of the partial derivatives of the log likelihood with respect to the model parameters.

\section{SIMULATION}

The method described in this paper for the maximum likelihood estimation of an aggregated Markov process has been implemented and tested extensively with a variety of models. We present here two examples for illustration. For these examples, the dwell time sequences were generated using a method described by Lauger (1988), which is based on the generation of exponentially distributed random numbers.

The program was written in $\mathrm{C}$ language, and the current executable version is available from the authors on request. The subroutines for variable metric optimization and matrix eigenvalue calculation are taken from Press et al. (1992). The calculations were done on an IBM RS/6000 Model 590, which is a 200 SPECfp computer, about four times faster than a Pentium 90.

\section{(a) Example 1}

The first example is based on the following model

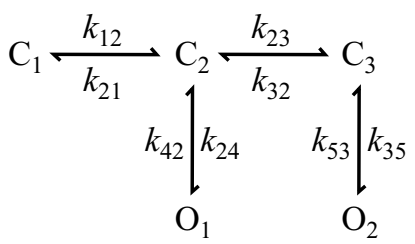

where $\mathrm{C}$ is closed and $\mathrm{O}$ open. Thus, there are two classes. This model was proposed as a gating mechanism for calcium-activated potassium channels in cultured rat muscle, as described by Magleby \& Pallotta (1983). The parameter values of the model are given in table 1 . A sequence of 5000 dwell times with a total duration of $18 \mathrm{~s}$ were simulated for analysis.

Parameter estimation results are presented in table 1. It can be seen that the estimated values are close to the underlying 'true' values employed to generate the data. Furthermore, the parameter error estimates are

\begin{tabular}{|c|c|c|c|}
\hline rate & $\begin{array}{l}\text { true } \\
\text { value }\end{array}$ & estimate & s.d. \\
\hline$k_{12}$ & 34 & 29 & 3 \\
\hline$k_{21}$ & 180 & 186 & 23 \\
\hline$k_{23}$ & 285 & 301 & 32 \\
\hline$k_{32}$ & 600 & 681 & 52 \\
\hline$k_{24}$ & 120 & 132 & 17 \\
\hline$k_{42}$ & 2860 & 3208 & 365 \\
\hline$k_{35}$ & 3950 & 3961 & 108 \\
\hline$k_{53}$ & 322 & 325 & 7 \\
\hline
\end{tabular}
small, the standard deviations being about $3-13 \%$ of the parameter values. This is not surprising because the

Table 1. Parameter estimates for example 1 



Figure 1. Sections of the likelihood surface for example 1. The sections are cut parallel to the parameter axes, and pass through the maximum point. The ordinate is the relative log likelihood. For abscissae, $k^{*}$ is the optimal value of the rate constant.

Table 2. Parameter estimates for example 2

\begin{tabular}{|c|c|c|c|}
\hline rate & $\begin{array}{l}\text { true } \\
\text { value }\end{array}$ & estimate & s.d. \\
\hline$k_{12}$ & 100 & 98 & 9 \\
\hline$k_{21}$ & 500 & 490 & 31 \\
\hline$k_{23}$ & 5000 & 5074 & 200 \\
\hline$k_{32}$ & 2000 & 1962 & 75 \\
\hline$k_{34}$ & 300 & 297 & 20 \\
\hline$k_{43}$ & 100 & 116 & 9 \\
\hline$k_{41}$ & 100 & 109 & 8 \\
\hline$k_{14}$ & 150 & 144 & 9 \\
\hline
\end{tabular}

data are perfect. What is noteworthy is the speed of the method. For this five state model and 5000 events, the program took $22 \mathrm{~s}$ to find a maximum, including ten iterations and 25 evaluations of the likelihood and its derivatives. The initial guesses of the parameters were their true values. Poor initial guesses may require a greater number of iterations, but not significantly. The program took typically 10-20 iterations to converge, even with grossly inaccurate starting points.

Figure 1 shows sections of the likelihood surface cut through the maximum point and parallel to the parameter axes. It is seen that the maximum is well defined along all axes. This is consistent with the calculated small error estimates. It is also seen that there is no local maximum point on these curves. We also tried the maximization by using different starting points and obtained the same final result, suggesting that the likelihood surface is smooth and has no local maxima. This is interesting since the likelihood is a complicated function of the model parameters, as seen from equation (8).

\section{(b) Example 2}

This is an example with a subconductance class. The model is a cyclic one of four states:

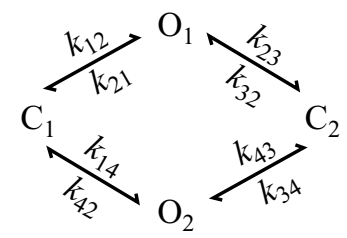

where $\mathrm{C}_{1}$ and $\mathrm{C}_{2}$ are closed, $\mathrm{O}_{1}$ is partly open, and $\mathrm{O}_{2}$ is fully open. The parameter values employed in the simulation are listed in table 2. A sequence of 5000 dwell times, totalling a duration of $5.4 \mathrm{~s}$, was simulated.

The model contains a loop. For a process at thermodynamic equilibrium, the rate constants around the loop must satisfy detailed balance, that is, the clockwise and anticlockwise products of the rate constants must be equal. To allow for these constraints 

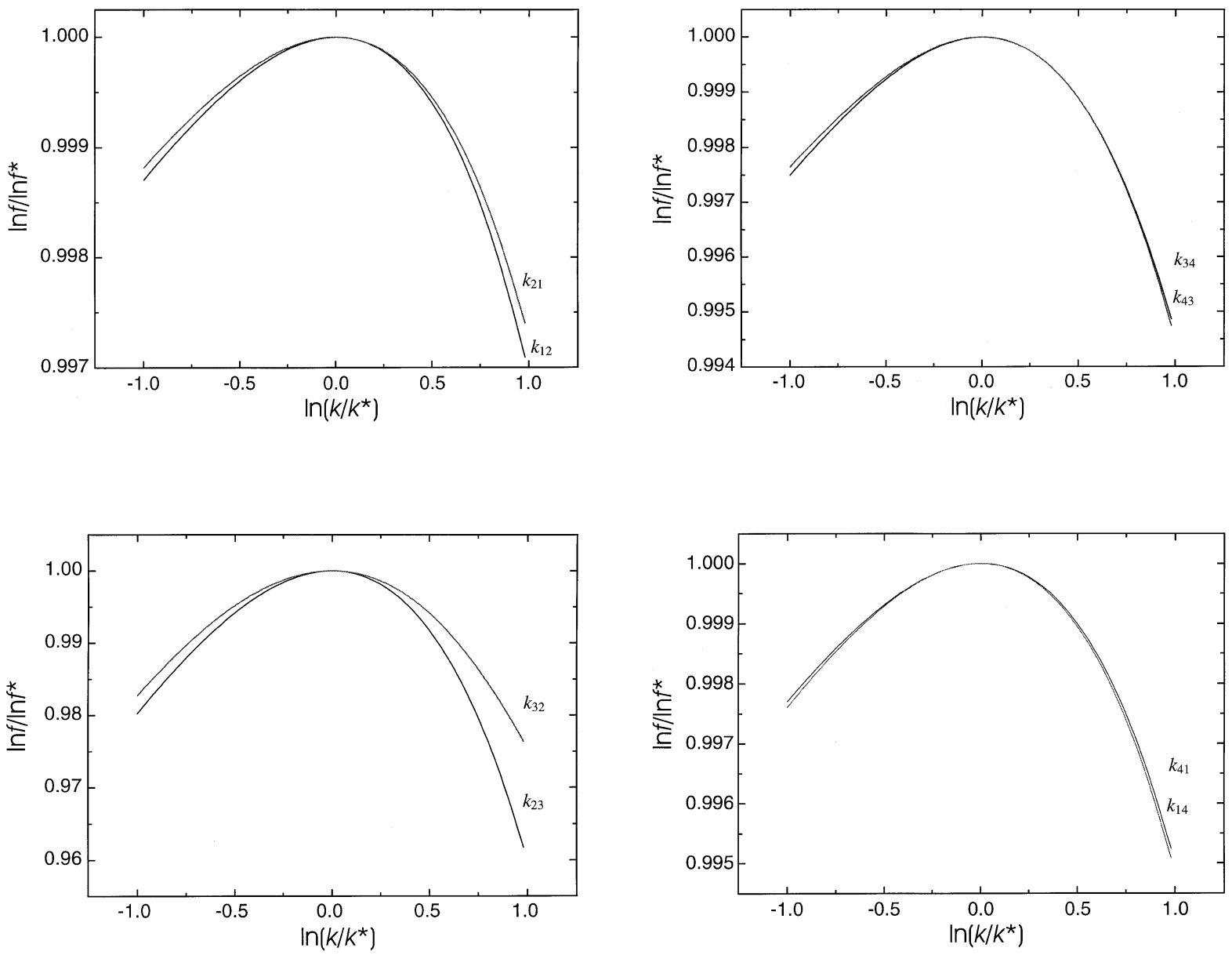

Figure 2. Sections of the likelihood surface for example 2. The sections are cut parallel to the parameter axes, and pass through the maximum point. The ordinate is the relative log likelihood. For abscissae, $k^{*}$ is the optimal value of the rate constant.

as well as for global fitting across different experimental conditions, e.g. concentration and voltage, in implementing the program we formulated the rate constants in an exponential form: $k_{i j}=C_{i j} \mathrm{e}^{a_{i j}+b_{i j} V}$, where $C_{i j}$ is the ligand concentration that affects $k_{i j}, V$ is the voltage, and $a_{i j}$ and $b_{i j}$ are new variables. For rates that are not scaled by any ligand, $C_{i j}=1$, and for rates that are voltage independent, $b_{i j}=0$. The detailed balance constraints, which are nonlinear in $k_{i j}$, become linear in the new variables $a_{i j}$ and $b_{i j}$. Such linear equality constraints may be incorporated into an unconstrained optimization procedure by making use of an elimination method (Fletcher 1981) to reduce the constrained problem into an unconstrained one. For a more detailed description of this technique, see Qin et al. (1996).

With the imposition of the detailed balance constraint, there are seven independent parameters to be estimated. The estimation results are shown in table 2. Again, the parameter estimates are very close to their true values, and the corresponding error estimates are small. Convergence occurred in $12 \mathrm{~s}$, taking nine iterations and 29 evaluations of likelihood and its derivatives.

As before, the maximization was repeated by varying the initial guesses of the parameters, and was shown to be robust. Examination of sections of the likelihood surface (figure 2) shows that the maximum is well defined and also confirms no local maxima.

\section{DISGUSSION}

We have described a maximum likelihood method for the modelling of aggregated Markov processes. The method employs a forward-backward recursive procedure for calculating the likelihood function and its derivatives. Maximization of the likelihood function is performed by a variable metric optimizer. Because analytical derivatives are used, the method is computationally efficient. It converges rapidly and is numerically stable.

Although the likelihood appears to be a complicated function of the model parameters, we have found empirically that the likelihood surface is relatively smooth and does not seem to involve local maxima for typical models. This makes it relatively easy to choose the initial guesses of parameters. In this context, it is interesting to notice similar findings reported by several other authors (Horn \& Lange 1983; Ball \& Sansom 1989). Nevertheless, caution must be taken and different initial guesses should be tried, especially for large and complicated models. We are planning to investigate the use of global optimization techniques to avoid local maximums. 
We have used the curvature of the likelihood function at the maximum point to estimate the standard errors of the model parameters. This approach assumes the likelihood surface is quadratic near the maximum point, which is approximately true in practice as shown in the examples. There are some other approaches for obtaining parameter error estimates, e.g. the segmentation method (Fredkin \& Rice 1992), the resampling method (Horn 1987) and the likelihood interval method (Auerbach 1993; Colquhoun \& Sigworth 1995). These approaches do not involve the quadratic assumption. Thus they may be used to obtain more accurate error estimates if the quadratic assumption was of doubtful reliability. These approaches have not been widely used in practice because of the intensive computation. Given the high speed of our algorithm, they could now become more accessible.

The current method assumes the availability of perfect data. This assumption fails in practice due to missed events arising from low-pass filtering and the presence of noise. The missed events lead to an overestimation of the observed dwell times and consequently adversely affect the estimation of model parameters. As shown by Qin et al. (1996), the current method can be partially corrected for the effects of missed events. That paper shows how reasonable parameter estimates can be derived from both synthesized data containing noise and subjected to lowpass filtering, and from patch-clamp data. An alternative correction for missed events is presented by Colquhoun et al. (1996), but that method requires more complicated computation.

Finally, we stress that the method can be applied to a wide class of problems, though we have confined our analysis to ion channel modelling. Possible applications include analysis of enzyme conformation changes (Radmacher et al. 1995), molecular motor movements (Finer et al. 1994), intensity fluctuations from single fluorophores (Basche et al. 1995) and capacitance changes of the cell membrane during synaptic vesicle fusion (Fernandez et al. 1984). Common to all these kinds of data are discrete steps of activity, a feature that is well suited for modelling via a Markov process.

We thank Dr J. Rice and Dr D. Fredkin for their comments on the manuscript. This work was supported by the National Science Foundation (9015986 and 9102233) and the Muscular Dystrophy Association and the NIH RR11114 (to F.S.).

\section{REFERENGES}

Auerbach, A. 1993 A statistical analysis of acetylcholine receptor activation in Xenopus myocytes: stepwise versus concerted models of gating. J. Physiol. 461, 339-378.

Ball, F. G. \& Sansom, M. S. P. 1988 Aggregated Markov processes incorporating time interval omission. Adv. Appl. Prob. 20, 546-572

Ball, F. G. \& Sansom, M. S. P. 1989 Ion-channel gating mechanisms: model identification and parameter estimation from single channel recordings. Proc. R. Soc. Lond. B 236, 385-416.
Basche, T., Kummer, S. \& Brauchle, G. 1995 Direct spectroscopic observation of quantum jumps of a single molecule. Nature, Lond. 373, 132-134.

Bellman, R. 1960 Introduction to matrix analysis. New York: McGraw-Hill.

Chay, T. R. 1988 Kinetic modeling for the channel gating process from single channel patch clamp data. J. Theor. Biol. 132, 449-468.

Colquhoun, D. \& Hawkes, A. G. 1982 On the stochastic properties of bursts of single ion channel openings and of clusters of bursts. Phil. Trans. R. Soc. Lond. B 300, 1-59.

Colquhoun, D. \& Hawkes, A. G. 1995 The principles of the stochastic interpretation of ion-channel mechanisms. In Single-channel recording, 2nd edn (ed. B. Sakmann \& E. Neher), pp. 397-482. New York: Plenum Press.

Colquhoun, D., Hawkes, A. G. \& Srodzinski, K. 1996 Joint distribution of apparent open times and shut times of single ion channels and maximum likelihood fitting of mechanisms. Phi. Trans. R. Soc. Lond. A 354, 2555-2590.

Colquhoun, D. \& Sigworth, F. J. 1995 Fitting and statistical analysis of single channel records. In Single-channel recording, 2nd edn (ed. B. Sakmann \& E. Neher), pp. 483-587. New York: Plenum Press.

Cox, D. R. \& Miller, H. D. 1965 The theory of stochastic processes. London: Methuen.

Fernandez, J. M., Neher, E. \& Gomperts, B. D. 1984 Capacitance measurements reveal stepwise fusion events in degranulating mast cells. Nature, Lond. 312, 453-455.

Finer, J. T., Simmons, R. M. \& Spudich, J. A. 1994 Single myosin molecule mechanics: piconewton forces and nanometre steps. Nature, Lond. 368, 113-119.

Fletcher, R. 1981 Practical methods of optimization. Chichester: John Wiley \& Sons.

Fredkin, D. R., Montal, M. \& Rice, J. A. 1985 Identification of aggregated Markovian models: application to the nicotinic acetylcholine receptor. In Proceedings of the Berkeley Conference in Honor of Jerzy Neymann and Jack Kiefer (ed. L. M. LeCam \& R. A. Olshen), pp. 269-289. Belmont, CA.

Fredkin, D. R. \& Rice, J. A. 1986 On aggregated Markov processes. J. Appl. Prob. 23, 208-214.

Fredkin, D. R. \& Rice, J. A. 1992 Maximum likelihood estimation and identification directly from single-channel recordings. Proc. R. Soc. Lond. B 239, 125-132.

Horn, R. 1987 Statistical methods for model discrimination. Biophys. J. 51, 255-263.

Horn, R. \& Lange, K. 1983 Estimating kinetic constants from single channel data. Biophys. J. 43, 207-223.

Lauger, P. 1988 Internal motions in proteins and gating kinetics of ionic channels. Biophys. J. 53, 877-884.

Magleby, K. L. \& Pallotta, B. S. 1983 Calcium dependence of open and shut interval distributions from calciumactivated potassium channels in cultured rat muscle. $J$. Physiol. 344, 585-604.

Press, W. H., Teukolsky, S. A., Vetterling, W. T. \& Flannery, B. P. 1992 Numerical recipes in C. Cambridge University Press.

Qin, F., Auerbach, A. \& Sachs, F. 1996 Estimating single channel kinetic parameters from idealized patch-clamp data containing missed events. Biophys. J. 70, 264-280.

Rabiner, L. R. 1989 A tutorial on hidden Markov models and selected applications in speech recognition. Proc. IEEE 77, 257-286.

Radmacher, M., Fritz, M. \& Hansma, H. G. 1995 Direct observation of enzyme activity with the atomic force microscope. Nature, Lond. 265, 1577-1579.

Received 21 October 1996; accepted 13 November 1996

As this paper exceeds the maximum length normally considered for publication in Proceedings B, the authors have agreed to make a contribution towards production costs. 